\title{
Developing an Online Examination APP System
}

\author{
Ting-Sheng Weng, Meng-Hui Hsu, and Der-Ching Yang
}

\begin{abstract}
The rapid development and prevalence of mobile communication technology has made mobile devices popular and indispensible tools in daily life. This study used Android Studio as the development environment, and used Java as the programming language to develop an online exam app. The proposed app allows users to test their learning effectiveness in mobile learning, and further improve self-learning ability.
\end{abstract}

Index Terms-APP, Android studio, cloud, online examination system, mobile learning.

\section{INTRODUCTION}

Mobile applications (mobile app, apps) allow smartphones and mobile devices to have diversified functions. The Ministry of Education of R.O.C. (2015) [1] has been promoting the Educational App Mall Project under the Education Cloud Alliance Plan, in order to develop e-learning allocation, and facilitate the adaptive, diversified, and mobile development of teaching.

Liao (2014) [2], in his "The effect of mobile learning on student learning achievement: A meta-analysis", mentioned that, in recent years, m-learning and Ubiquitous Learning have attracted much attention in the field of information education. Moreover, some studies have verified that the benefits of m-learning on students' learning motivation, learning attitude, and learning achievement exceed those of traditional teaching methods [3], while some studies indicated that the influence of m-learning on improving students' learning attitude or achievement is not significantly better than that of traditional teaching.

A cloud app can be applied to learning methods, such as Massive Open Online Courses (MOOCs) and afterschool assisted learning, which may be beneficial to learners of different levels and needs.

\section{ReseARCh Motives AND PURPOSES}

Due to the prevalence of mobile devices and the internet, users can access online learning systems at anywhere and anytime. They thus can determine the time and place of learning, engage in self-online learning, and assess learning

Manuscript received April 14, 2016; revised June 18, 2016.

Ting-Sheng Weng is with the Department of Business Administration at National Chiayi University, Chiayi City 600, Taiwan R.O.C (e-mail: politeweng@mail.ncyu.edu.tw).

Meng-Hui Hsu is with the Department of Mechanical Engineering, Kun Shan University, No.195, Kunda Rd., Yongkang Dist., Tainan City 710, Taiwan R.O.C. (e-mail: mhhsu@mail.ksu.edu.tw).

Der-Ching Yang is with the Graduate Institute of .Mathematics and Science Education at National Chiayi University, Minsyong Township, Chiayi County 621, Taiwan R.O.C (e-mail: dcyang@mail.ncyu.edu.tw). effectiveness conveniently.

\section{LITERATURE REVIEW}

The convenience of information acquisition and sharing brought by m-learning, users can use mobile devices and e-learning systems to acquire learning assistance or supplementary data, or even test their own progress. In 2013, the Ministry of Education of the R.O.C. [4] implemented the M-learning Tutoring Program, with the aim to use m-learning to achieve life-based and self-paced learning.

In "Integration of mobile intelligence with technological marketing application system", Lin (2012) [5] mentioned that smartphone users possess the consumer characteristic of being able to acquire instant information at anytime and anyplace. Hence, warehouse stores have started to develop apps for online promotional materials, and launched digital marketing campaigns using QR codes. Chen (2013) [6] explored the use of cloud medical app on the integrated home care for patients with moderate to severe cirrhosis, and developed a cloud care management app according to the needs of patients, family members, and nurses. The app is able to reduce the readmission rate and decrease the waste of medical resources. Based on the cross-enterprise integration perspective, Lin (2014) [7] adopted the innovation diffusion theory to discuss the use of mobile banking apps in business promotion. Shih (2015) [8] experimented flipped afterschool tutoring on schoolchildren, and used the game-based diagnosis to assess the pretests on the use of apps on mathematics tutoring.

Own (2013) [9] integrated mobile communication and app design learning models in an online course, and used online exams to test learning outcomes. He also performed statistical analysis regarding how the differences in students' learning characteristics lead to differences in the use of different learning devices. The questionnaire survey results showed that, $89.47 \%$ of the students using smartphones to engage in learning perceived less learning stress, and $80.26 \%$ suggested that the use of smartphones is very beneficial to their learning. Lin et al. (2013) [10] conducted a study on a video Q\&A platform of an interactive cloud community and app design. By using community sharing, their design solved the problems that could not be explained by text description. Users' knowledge is pooled to provide answers to questions in everyday life. The app also has search functions for convenience of usage. Yang and Huang (2015) [11] investigated the application of situational learning to elementary school natural science courses. They integrated real situations with the $\mathrm{m}$-learning to investigate learners' learning motivation, attitude, and effectiveness during natural exploration. They combined cloud computing with a handheld device app, and integrated inquiry-based learning strategies to develop an inquiry-based situational learning model. They observed the 
influence of the app on learners' learning motivation, attitude, and effectiveness of natural exploration.

It is clear that with the advancement of mobile information technologies, mobile digital learning has changed the teaching, learning, and practices of education.

\section{ONLINE EXAMINATION APP}

\section{A. Android Studio}

Android Studio is a new IntelliJ IDEA-based development environment released by Google for Android developers at the Google I/O 2013 Developer Conference. It has the advantages of simplified and optimized development workflow, and can provide cross-platform support for Windows, Mac OS X, and Linux operating systems.

The old version of the Android development environment is Eclipse IDE, and the officially released plug-in program used in combination with Eclipse IDE is called ADT
(Android Development Tools Plugin). The ADT plugin program needs to be installed in Eclipse IDE to provide an open interface for related programs. As ADT is no longer updated, this study used Android Studio as the development environment to develop the program.

\section{B. Development Environment}

The Android development environment is Windows 7, 64-bit, and JDK8, which were installed prior to developing the app.

\section{Programming Language and XML}

Android uses Java language for programming, and supports the Java SE syntax and Java API libraries. XML is used to define the resources and user interface of the Android app. The declaration method is mainly used, and the interface components, language system, background, and layout can be developed without writing a line of Java program codes.

TABLE I: THE EXAMPLE OF JAVA PROGRAM

\begin{tabular}{|c|c|}
\hline package com.example.administrator.test3; & Develop the package by adding instructions and statements to the package \\
\hline & Import the category in the package \\
\hline import android.support.v7.app.ActionBarActivity; & Import ActionBarActivity \\
\hline import android.os.Bundle; & Import android.os.Bundle package \\
\hline import android.view.View; & Import android.view.View package \\
\hline import android.widget.RadioButton; & Import android.widget.RadioButton package \\
\hline import android.widget.RadioGroup; & Import android.widget.RadioGroup package \\
\hline import android.widget.TextView; & Import android.Widget.TextView package. \\
\hline \multicolumn{2}{|l|}{ Extended from Activity } \\
\hline public class MainActivity extends ActionBarActivity \{ & MainActivity extends ActionBarActivity \\
\hline private TextView output; & Text View text component variable output \\
\hline private RadioGroup radioGroup; & Option button component variable radio Group \\
\hline private RadioButton rd1, rd2, rd3; & Option button components rd1, rd2, and rd3 \\
\hline @Override & Method for overriding on Create of Activity \\
\hline \multicolumn{2}{|l|}{ protected void onCreate(Bundle savedInstanceState) \{} \\
\hline super.onCreate(savedInstanceState); & Description of method for inclusion of parent category on Create \\
\hline setContentView(R.layout.activity_main); & Designate the picture configuration resources used by this component \\
\hline output = (TextView) findViewById(R.id.textView2); & Obtain the value of textView2 \\
\hline radioGroup = (RadioGroup) findViewById(R.id.rgp); & Obtain the value of rgp \\
\hline rd1 = (RadioButton) findViewById(R.id.rb1); & Obtain the value of rb1 \\
\hline rd2 = (RadioButton) findViewById(R.id.rb2); & Obtain the value of rb2 \\
\hline rd3 = (RadioButton) findViewById(R.id.rb3); & Obtain the value of rb3 \\
\hline $\begin{array}{l}\text { radioGroup.setOnCheckedChangeListener(listener); } \\
\text { \} }\end{array}$ & Register the object of listener \\
\hline $\begin{array}{c}\text { private RadioGroup.OnCheckedChangeListener listener = } \\
\text { new RadioGroup.OnCheckedChangeListener() \{ }\end{array}$ & When the user changes option, listener of interface will be used for processing \\
\hline 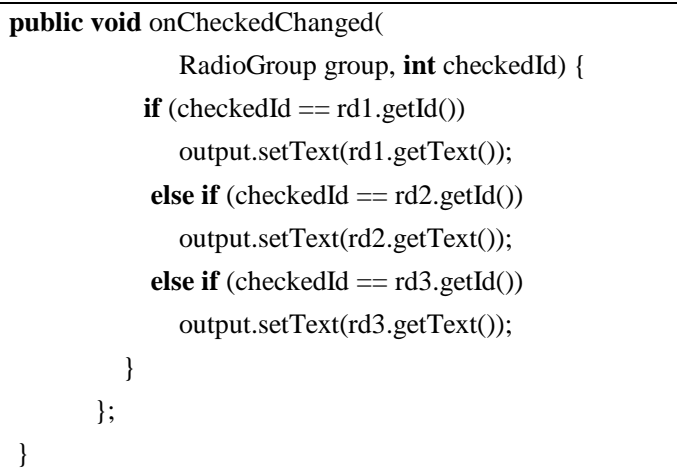 & After the user changes an option, the chosen name will be displayed in the place of output \\
\hline
\end{tabular}




\section{Java Program}

The example of Java program is given in Table I, as follows:

\section{E. APP Development Procedures}

The flow chart of the app development is as shown in Fig. 1.

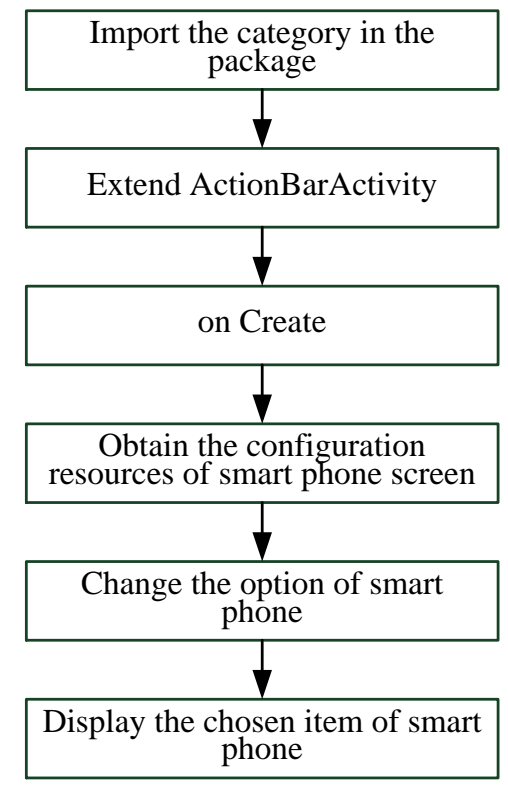

Fig. 1. Flow chart of development of smart phone APP.

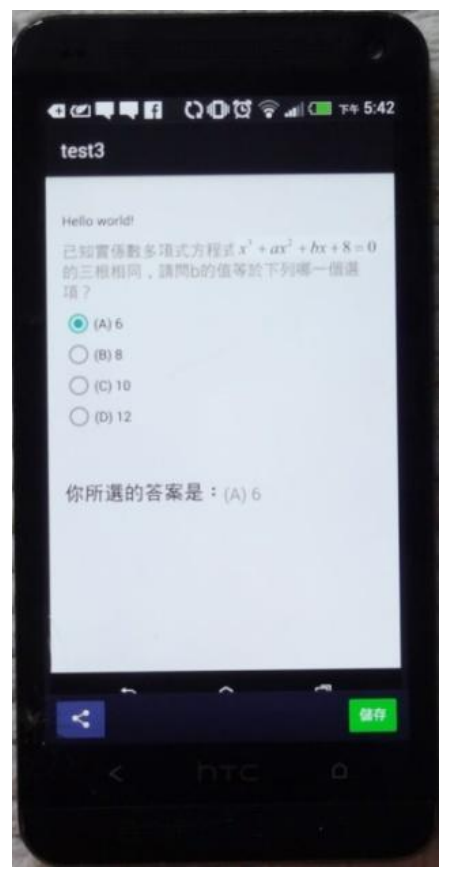

Fig. 2. Screen of APP Online Examination.

The app imports the category required in the Android package, and the programming codes are extended to extend the existing category. As a smartphone interface is written using objects, once the screen configuration is ready, the names of various components must be stored in variables. When the user changes a chosen item, the item chosen by the user is then displayed in the content.

As shown in Fig. 2. Taking the test items of polynomial equation for example: it is known that the three roots of polynomial equation with real coefficients $x^{3}+a x^{2}+b x+8=0$ are the same, which of the following is equal to the value of $b$ ? (A)6; (B) 8 ; (C)10; (D)12. If (A)6 is chosen, the screen will display: The answer you chose (A)6 is wrong, the right one is (D) 12 .

Due to technological advancement and the convenience of mobile devices, smartphones have become indispensible in people's life, and the usage rate of app has been increasingly. Unlike paper-based self-exams, the online exam app developed by this study uses the existing programming technology and development environment to develop an app with high practicability. Users can easily conduct self-test, thus achieving the effect of self-learning and self-motivation.

\section{CONCLUSIONS}

This study used Android Studio as the development environment, and used Java language for programming. The developed online exam app can be used on smartphones and tablets. Users can take instant exams at anytime and anyplace, immediately know their learning, and identify areas of improvement. The exams are also available for many subjects. With the growing popularity of MOOCs, the proposed app can be widely used as a part of teaching materials, and serve as am efficient tool for interactive learning and assessments.

\section{SUGGESTIONS}

Future studies can define the scope of exam items, in order to immediately determine the level of item difficulty. The functions of batch import, batch export, group test, and verification should be developed. Along with a diagnostic system, the app can instantly analyze the answers, and present diagnostic reports for the exam results. With exams at different levels, the app can serve as an effective tool in remedial teaching.

\section{ACKNOWLEDGMENT}

The authors appreciate the comments of the reviewers. This project was supported by the Ministry of Science and Technology No. MOST 104-2511-S-415-008.

\section{REFERENCES}

[1] Ministry of Education, "2015 education cloud strategic alliance plan -2015 national teaching APP market, joy of teaching application, and implementation plan of teaching micro-film recruitment activity".

[2] Y. K. Liao, "The effect of mobile learning on student learning achievement: A meta-analysis," Ministry of Science and Technology project, MOST103-2410-H034-039.

[3] Z. Y. Own, "A study on application of mobile learning model to chemistry education," Chemistry Education in Taiwan, vol. 1, no. 1 , pp. 86-91, May 2014.

[4] Ministry of Education, "M-learning tutoring program for senior (vocational) high school".

[5] Y. M. Lin, "Integration of mobile intelligence with technological marketing application system".

[6] S. T. Chen, "A preliminary exploration on application of medical app to home care for patients with moderate to severe cirrhosis," Department of Health, Executive Yuan project, DOH102-HO-1089.

[7] W. R. Lin, "The application study of multi-enterprise integrated perspective for innovation diffusion theory in mobile banking app service system implementing strategy," National Science Council project, NSC102-2410-H034-028. 
[8] W. C. Shih, "Inverted tutoring: A study of using joyful app of diagnostic assessment in pre-tests of math tutoring," Ministry of Science and Technology project, MOST103-2511-S468-003.

[9] Z. Y. Own, "The difference of students' character apply in various learning devices - A example of university chemistry general course," National Science Council project, NSC101-2511-S126-005.

[10] P. C. Lin, J. F. Wang, M. H. Lin, J. H. He, and G. L. Huang, "iAsking - An interactive multimedia Q\&amp; A platform and APP design with cloud social networking," National Science Council Project, NSC101-2218-E272-002.

[11] S. Yang and I. Huang, "Subproject 4: The application and evaluation of integrating cloud computing and handheld-device app in inquiry situated learning for elementary school natural science courses," National Science Council project, NSC101-2511-S008-011-MY3.

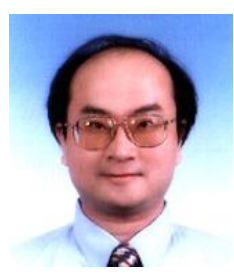

Ting Sheng Weng is an associate professor of the Department of Business Administration at National Chiayi University, Taiwan. He received his Ph.D. in division of management and information science, Graduate School of Advanced Technology from Kinki University, Japan in 2000. He had served as visiting professor at Meiji University in Japan from 2006 through 2007. His major research interests include science technology and management, e-learning, information education, technology education, MIS, and image processing.

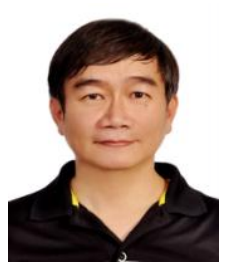

Meng-Hui Hsu took the M.S. and Ph.D. from the Department of Mechanical Engineering, National Cheng-Kung University, Taiwan, 1990 and 1996, respectively. Now Dr. Hsu is an associate professor of Mechanical Engineering Department at Kun-Shan University in TAIWAN. His research interests include mechanism and machine design, theory of creative design, and servo control systems.

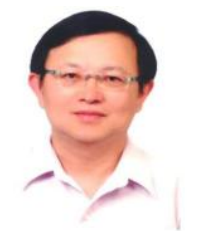

Der-Ching Yang is a professor of the Graduate Institute of .Mathematics and Science Education at National Chiayi University. Dr. Yang received his $\mathrm{PhD}$ in mathematics Education from University of Missouri-Columbia in 1995. His research focuses on number sense, mathematics teaching \& learning, technology \& mathematics teaching and learning, curriculum study. 